\title{
Enhancement and localization of electric field in dielectric photonic crystal sandwiched between two Bragg-mirrors
}

\author{
Naim Ben Ali ${ }^{1,2, *}$, Abdulaziz Salem Alghamdi ${ }^{1}$, Issam Badawi ${ }^{1}$, Mounir Kanzari ${ }^{2}$ \\ ${ }^{1}$ College of Engineering, Industrial Engineering Department, Haïl University, 2440-Haïl City, Saudi Arabia \\ 2Photovoltaic and Semiconductor Materials Laboratory, El-Manar University-ENIT PO Box 37, Le belvedere 1002-Tunis, Tunisia
}

\section{A R T I C L E I N F O}

\section{Article history:}

Received 12 June 2016

Received in revised form

25 August 2016

Accepted 18 September 2016

\section{Keywords:}

Photonic

Optic

Light localization

Hybrid structure

Electric field

\section{Introduction}

Photonic crystals (PC) are a class of artificial periodic composite materials which exhibit electromagnetic band gap (i.e. ranges of frequency in which light cannot propagate through the structure). Recently, PCs are undergoing a rapid development. Being their ability to control electromagnetic wave propagation, many potential applications are proposed (Joannopoulos et al., 1995; Johnson and Joannopoulos, 2002; Topolancik et al., 2003; Loncar et al., 2003). If a linear defect is incorporated into a crystal, propagating modes confined within the defect can be created for frequencies within the photonic band-gap. The simplest form of PCs is the one-dimensional $(1 D)$ periodic structure. It consists of a stack of alternating layers having a low (L) and high $(\mathrm{H})$ refractive indices. When whose (layers) thicknesses satisfy this condition (Kittel, 1976): $n_{L} d_{L}=n_{H} d_{H}=\lambda_{0} / 4\left(\lambda_{0}\right.$ is the reference wavelength), PC is known as Bragg Mirror (Kittel, 1976). These materials (PCs) have important technological applications - in the development of micro-lasers and optical transistors, for example - because they can coherently localize light when disorder is introduced (either by structural defects or by doping the material with resonant atoms or molecules) (Sajeev, 1997).

\footnotetext{
* Corresponding Author.

Email Address: naimgi2@yahoo.fr (N. B. Ali)

https://doi.org/10.21833/ijaas.2016.09.007

2313-626X/C) 2016 The Authors. Published by IASE

This is an open access article under the CC BY-NC-ND license

(http://creativecommons.org/licenses/by-nc-nd/4.0/)
}

Light localization is a fundamental optical phenomenon arising from strong resonant scattering and interference effects (Wiersma et al., 1997). Also PC cavities can localize light into nano-scale volumes with high quality factors (Jelena et al., 2006). This permits a strong interaction between light and matter, which is important for the construction of classical light sources with improved properties (e.g., low reshold lasers) and of non-classical light sources (such as single and entangled photon sources) that are crucial pieces of hardware of quantum information processing systems (Jelena et al., 2006).

The specific structure that we investigated is a hybrid photonic structure formed by the combination of two Bragg mirrors with a photonic crystal (Fig. 1). We demonstrate that strong localization of light can be achieved in PC sandwiched between two Bragg mirrors. The possibilities of using this phenomenon in visible spectral range open a way toward the optical memory devices and increase the efficiency of nonlinear optical interactions and if one day the light will be totally confined inside theses crystals it would be possible to make optical- memories (using photons) with large storage capacity and without the current problems of overheating (Lannebère and Silveirinha, 2015) of electronic-memories (which use electrons) (Trofimov et al., 2003).

The rest of the paper is organized as follow: In section 2, we introduce the method used in this study (Transfer Matrix Method) to calculate the electric field intensity in 1D hybrid Bragg mirrors/photonic crystal (PC). Next we present the simulation results in section 3 and we conclude the paper in the section 4 . 


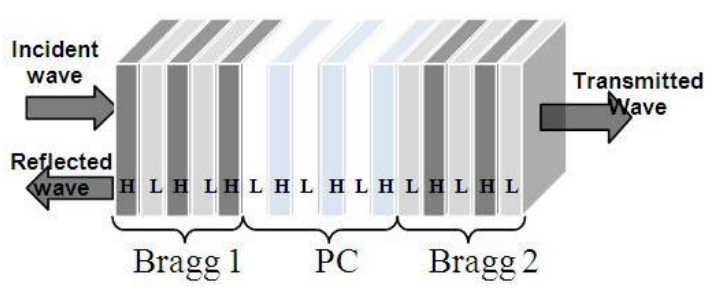

Fig. 1: Schematic representation showing the geometry of the hybrid photonic structure formed by a photonic crystal sandwiched between two Bragg mirrors

\section{Mathematical theory}

\subsection{Transfer matrix method (TMM)}

The method for calculating the electric field intensity in photonic crystals is the transfer matrix method (TMM), it consists of the calculation of the forward $E^{+}$and the backward $E^{-}$propagating electric field components. This method is introduced by Yeh (Yeh and Yariv, 1984) and it's the most used method and easiest to study the propagation of waves in multilayer structures. It permits particularly to extract and solve the standard problem of the photonic band structures (transmission, reflection, absorption and electric field distribution) spectrum. For stratified layers within m layer, the amplitudes of the electric fields of incident wave $E_{0}^{+}$, reflected wave $E_{0}^{-}$and transmitted wave $E_{m+1}^{+}$after $m$ layers can be related via the following matrix (Yeh and Yariv, 1984) (Eq. 1):

$$
\left(\begin{array}{l}
E_{0}^{+} \\
E_{0}^{-}
\end{array}\right)=C_{1} C_{2} C_{3} \ldots C_{m+1}\left(\begin{array}{l}
E_{m+1}^{+} \\
E_{m+1}^{-}
\end{array}\right)
$$

Here $C_{j}$ is the product of the propagation matrix $C_{p r}$ and the interface Matrix $C_{\text {int }}$ with the matrix elements (Eqs. 2 and 3):

$$
\begin{aligned}
C_{p r} & =\left(\begin{array}{cc}
e^{i \varphi_{j-1}} & 0 \\
0 & e^{-i \varphi_{j-1}}
\end{array}\right) \\
C_{i n t} & =\frac{1}{t_{j}}\left(\begin{array}{cc}
1 & r_{j} \\
r_{j} & 1
\end{array}\right)
\end{aligned}
$$

where, $t_{j}$ and $r_{j}$ are the Fresnel transmission and reflection coefficients, respectively, between the $j-1^{\text {th }}$ and $j^{\text {th }}$ layer (Yeh and Yariv, 1984).

\subsection{Calculation of the electric field intensity throughout a multilayer structure}

The following derivations illustrate how the absolute square of the electric field can be calculated throughout a multilayer structure. From this point on, it will be referred to as the intensity. Given an arbitrary input field, the main interest is in calculating the normalized intensity. For example, given an input field $E_{1}^{+}$, the normalized intensity at an interface $i$ of a multilayer structure is $\frac{\left|E_{i}\right|^{2}}{\left|E_{1}^{+}\right|^{2}}$ (Cheng, 1993).
The aim of this section is to derive an expression for $E_{i}$ from which the normalized intensity can be calculated (Cheng, 1993). Referring to Fig. 2, the field at the interface 1 can be related to that of the interface 2 using this matrix (Eq. 4):

$\left(\begin{array}{l}E_{1}^{+} \\ E_{1}^{-}\end{array}\right)=\frac{1}{t_{12}}\left(\begin{array}{cc}1 & r_{12} \\ r_{12} & 1\end{array}\right)\left(\begin{array}{cc}\exp (j k l) & o \\ 0 & \exp (-j k l)\end{array}\right)\left(\begin{array}{l}E_{2}^{+} \\ E_{2}^{-}\end{array}\right)$

where, $k=\frac{2 \pi}{\lambda} n_{2}$. Multiplying this expression out gives: Generally, for any two interfaces with a distance $L$ separating them and $k=\frac{2 \pi}{\lambda} n$, with $n$ representing the refractive index of the material separating the two interfaces, the expression for the forward electric field is given by (Cheng, 1993) (Eqs. 5 and 6):

$$
E_{I+1}^{+}=\frac{1-\Gamma_{i} r_{i(i+1)}}{\exp (j k L)\left(1-r_{i(i+1)}\right.} E_{i}^{+}
$$

with

$$
\Gamma_{m}=r_{m(m+1)}
$$

Therefore, for a known incident forward field, provided that $\Gamma$ has been calculated using the TMM (Yeh and Yariv, 1984), the intensity can be calculated at any interface of a multilayer structure by propagating the electric field forwards layer by layer using Eq. 5 (Cheng, 1993).

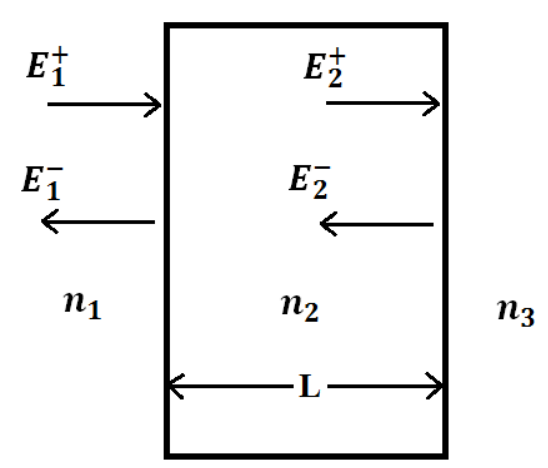

Fig. 2: Diagram of two interfaces separated by a thickness $L$

\section{Results and discussions}

By using the TMM to extract the electric field intensity in $1 D$ hybrid Bragg mirrors/photonic crystal (Fig. 1) in the visible frequency spectral range, we show that the corresponding electric field intensity exhibits interesting properties. So in this paper we search to localize and enhance the electric field in the middle structure i.e. the photonic crystal. Here we assume that the front and the back media have refractive index $n_{0}=1$ (index of air). In addition, we have chosen the high $(H)$ refractive index $\mathrm{n}_{\mathrm{H}}$ and the low $(L)$ refractive index $\mathrm{n}_{\mathrm{L}}$ of BMs and $\mathrm{PC}$ by combining Air, Ceramic, $\mathrm{TiO}_{2}, \mathrm{SiO}_{2}$ and Roger, with refractive indices $n_{\text {Air }}=1, \quad n_{\text {Ceramic }}=3, \quad n_{\mathrm{TiO} 2}=2.3$, 
$n_{\mathrm{SiO} 2}=1.45$ and $n_{\text {Roger }}=3.134$, respectively. We assume, all the regions to be linear, homogenous, nonabsorbing. We demonstrate that the electric field intensity depends on the refractive indices, the number and the thicknesses of layers, which construct the hybrid structure. In addition the electric field intensities are calculated for the resonance peak wavelength at the edge of the wide PBG quoted small wavelengths.

At the present we start our study by the representation of the transmission of light in a periodic photonic crystal constituted by the alternation of 48 layers of Ceramic and $\mathrm{TiO}_{2}$ (Fig. 3). So, Fig. 4 represents the electric field intensity in this photonic structure for the resonance peak wavelength $\lambda=0.456 \mu \mathrm{m}$. The vertical lines represent the interfaces between the successive layers and it is clear that the electric field is distributed in all layers of the structure as well as their intensity is very weak. By varying some parameters of the hybrid photonic system constructed by using periodic photonic crystal sandwiched between two Bragg mirrors, we can confine, localize and enhance the electric field in the photonic crystal.

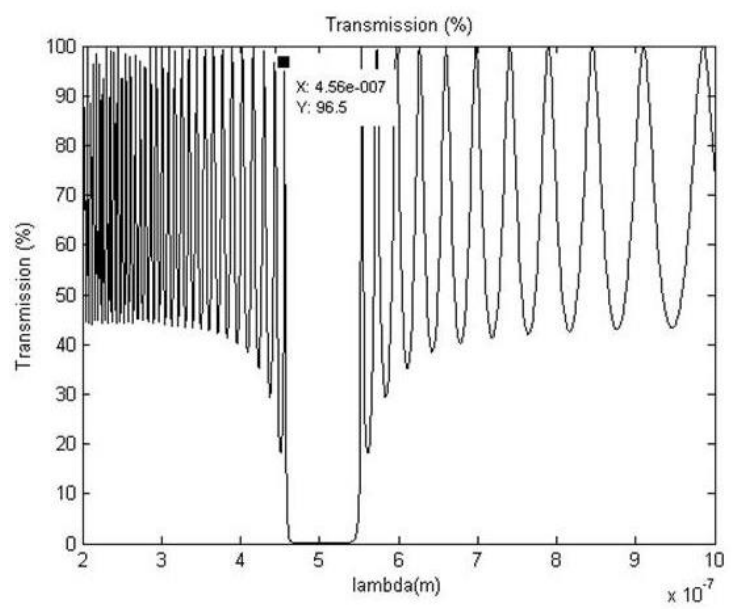

Fig. 3: Variation of the transmittance versus the angular frequency for a periodic multilayered system (48 layers with $\mathrm{n}_{\mathrm{H}}=3$ and $\mathrm{n}_{\mathrm{L}}=2.3$ )

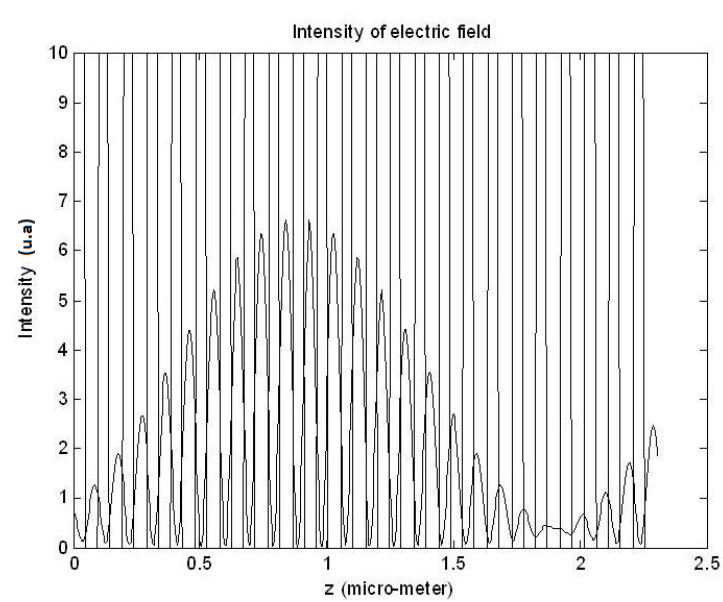

Fig. 4: Variation of the electric field intensity, versus the thickness of periodic photonic structure (48 layers, $\mathrm{n}_{\mathrm{H}}=3$ and $\mathrm{n}_{\mathrm{L}}=2.3$ )

\subsection{Effects of the refractive indices}

In this section we start with the study of the effects of the refractive indices constituting the Bragg mirrors and the $\mathrm{PC}$, on localization and enhancement of electric field through the PC. The thicknesses of the two materials that constitute the BMs have been chosen to satisfy the Bragg condition: $n_{L} d_{L}=n_{H} d_{H}=\lambda_{0} / 4$ and the thicknesses of the two materials that constitute the PC have been chosen to satisfy the condition: $n_{L} d_{L}=n_{H} d_{H}=\lambda_{0} / 2$, where $\lambda_{0}=0.5$ ? $m$ is the reference wavelength. The number of layers of every BM and the PC is 16 , so the hybrid structure is constituted by 48 layers. For the first BM we chose $\mathrm{SiO}_{2}(\mathrm{~L})$ and $\mathrm{TiO}_{2}(\mathrm{H})$ as two elementary layers, with refractive indices $n_{L}=1.45$ and $n_{H}=2.3$, respectively. For the $\mathrm{PC}$ we chose $\mathrm{Air}(\mathrm{L})$ and $\mathrm{TiO}_{2}$ (H) as two elementary layers $\left(n_{L}=1\right.$ and $\left.n_{H}=2.3\right)$ and the second BM is constituted by alternating Roger $(\mathrm{H})$ and $\mathrm{SiO}_{2}(\mathrm{~L})$ with refractive indices $n_{L}=$ 1.45 and $n_{H}=3.134$. Fig. 5 shows the transmission of light through this hybrid photonic structure and Fig. 6 shows the distribution of the electric field intensity through this hybrid structure. The vertical lines represent the interfaces between the successive layers. It is clear that the electric field is localized in the middle structure (i.e. the PC) and it is very weak in the two Bragg mirrors. In addition, by the Fig. 6 we conclude that the electric field intensity is eight times superior to that of given by the periodic PC (Fig. 4).

Now if we replace the $\mathrm{SiO}_{2}$ in the second $\mathrm{BM}$ by the Ceramic (i.e. we decrease the contrast of refractive index), the electric field intensity becomes more important in the PC (Fig. 7). For the Bragg_1 mirror, if we replace the high refractive index material $\left(\mathrm{TiO}_{2}\right)$ by the Ceramic one, the electric field intensity is increased and enhanced in the PC (Fig. 8 ). Now we change the Ceramic by the Roger and the $\mathrm{SiO}_{2}$ by the Air in the Bragg_1 mirror. The intensity increased in the PC and is reduced in the two Bragg mirrors (Fig. 9). It is clear that any change or variation of the refractive indices of the PC leads to a decrease of the electric field intensity in the middle photonic structure (PC) and it's an increase in the two Bragg mirrors. Therefore we notice that if we chose for the first BM Roger and Air as two elementary layers, for the PC Air and $\mathrm{TiO}_{2}$ and for the second BM Roger and Ceramic as two elementary layers we obtain the maximal value of electric field intensity (Fig. 9). So, it is clear if we increase the contrast of refractive index of the first Bragg mirror (Bragg_1) and we decrease the one of the second Bragg mirror (Bragg_2), we enhance (i.e. the value of electric field intensity is increased) and localize the electric field in the PC.

\subsection{Effects of the number of layers}

In this part we study the effect on the electric field intensity of the layers number of each structure that constitutes the hybrid photonic system (Fig. 9). With the increase of the number of layers of the PC 
we notice an increase of the intensity of the electric field in the PC and in the Bragg mirrors. So, since we try to confine the light only in the PC we have noted that when the number of layers of the PC attains 32 , the electric field intensity increases in the PC and decreases in the BMs with low oscillations. Two envelop oscillations of the electric field intensity were found (Fig. 10 (a)). In the last case, only one envelops oscillation is observed (Fig. 9).

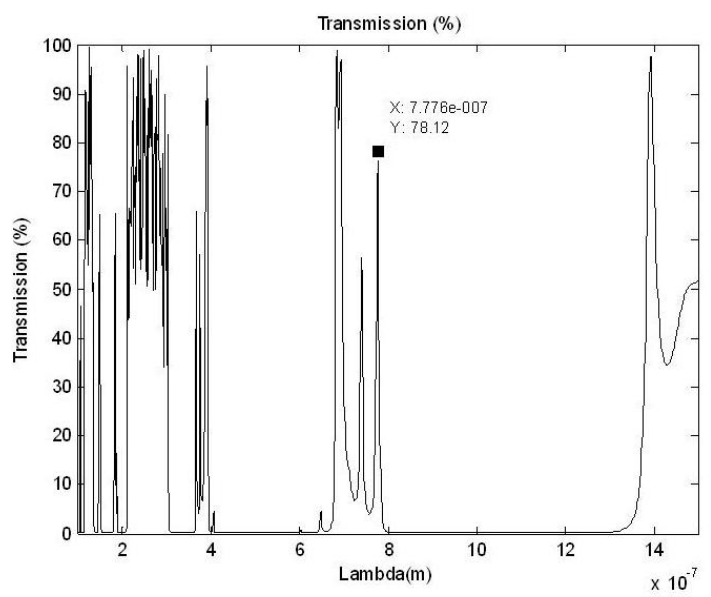

Fig. 5: Variation of the transmittance, versus the wavelength $(\mathrm{m})$ for hybrid photonic structure [Bragg_1: 16 layers $\left(\mathrm{n}_{\mathrm{H}}=2.3\right.$ and $\left.\mathrm{n}_{\mathrm{L}}=1.45\right) / C P: 16$ layers $\left(\mathrm{n}_{\mathrm{H}}=2.3\right.$ and $\left.n_{L}=1\right),\left(\lambda_{0} / 2\right) /$ Bragg_2: 16 layers $\left(n_{H}=3.134\right.$ and $\left.n_{L}=1.45\right]$

However the electric field intensity increases in the Bragg mirrors for the number of layers greater than 32 layers and it decreases for the number of layers equal to 48 layers. In this last case, three envelop oscillations of the electric field intensity were observed in the PC (Fig. 10 (b)). Moreover the number of the envelop oscillations increases by increasing the number of layers as shown in (Fig. 10 (c)). Indeed this last figure shows the presence of four envelop oscillations in the case of 64 layers of the PC. In conclusion the multilayer hybrid structure Bragg_1/ (PC) $)^{\mathrm{N}} /$ Bragg_2 exhibits N envelop oscillations of the electric field intensity.

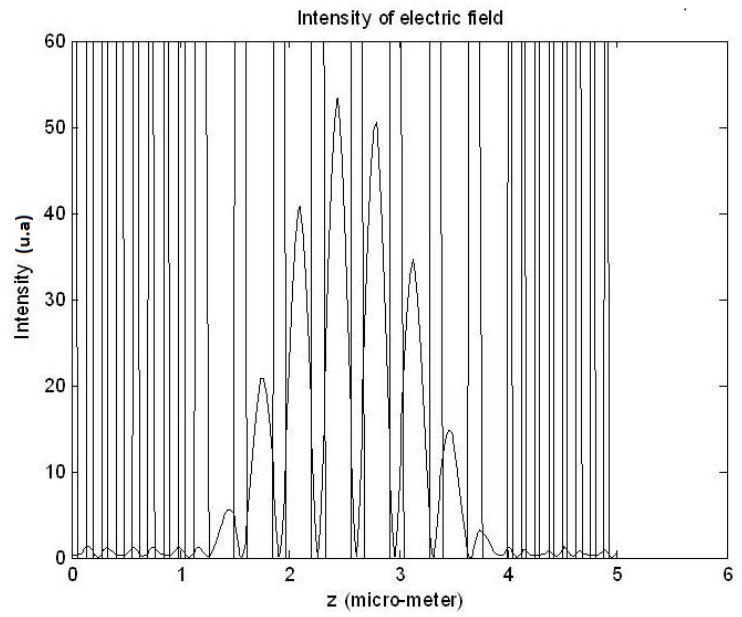

Fig. 6: Variation of the electric field intensity, versus the thickness $(\mu \mathrm{m})$ of hybrid photonic structure [Bragg_1: 16 layers $\left(\mathrm{n}_{\mathrm{H}}=2.3\right.$ and $\left.\mathrm{n}_{\mathrm{L}}=1.45\right) / C P: 16$ layers $\left(\mathrm{n}_{\mathrm{H}}=2.3\right.$ and $\left.\mathrm{n}_{\mathrm{L}}=1\right),\left(\lambda_{0} / 2\right) /$ Bragg_2: 16 layers $\left(\mathrm{n}_{\mathrm{H}}=3.134\right.$ and $\left.\mathrm{n}_{\mathrm{L}}=1.45\right]$

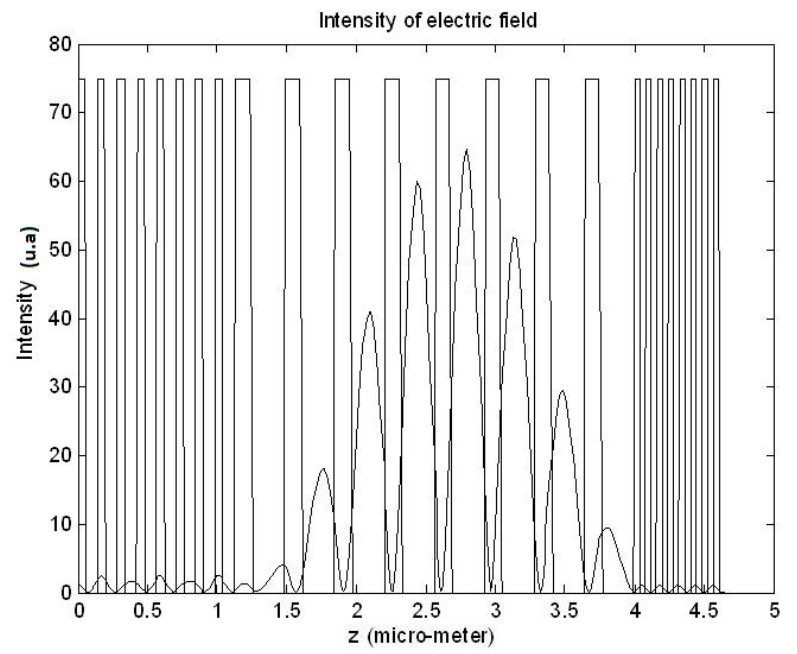

Fig. 7: Variation of the electric field intensity, versus the thickness $(\mu \mathrm{m})$ of hybrid photonic structure [Bragg_1: 16 layers $\left(\mathrm{n}_{\mathrm{H}}=2.3\right.$ and $\left.\mathrm{n}_{\mathrm{L}}=1.45\right) / \mathrm{CP}: 16$ layers $\left(\mathrm{n}_{\mathrm{H}}=2.3\right.$ and $\left.\mathrm{n}_{\mathrm{L}}=1\right),\left(\lambda_{0} / 2\right) /$ Bragg_2: 16 layers $\left(\mathrm{n}_{\mathrm{H}}=3.134\right.$ and $\left.\mathrm{n}_{\mathrm{L}}=3\right]$

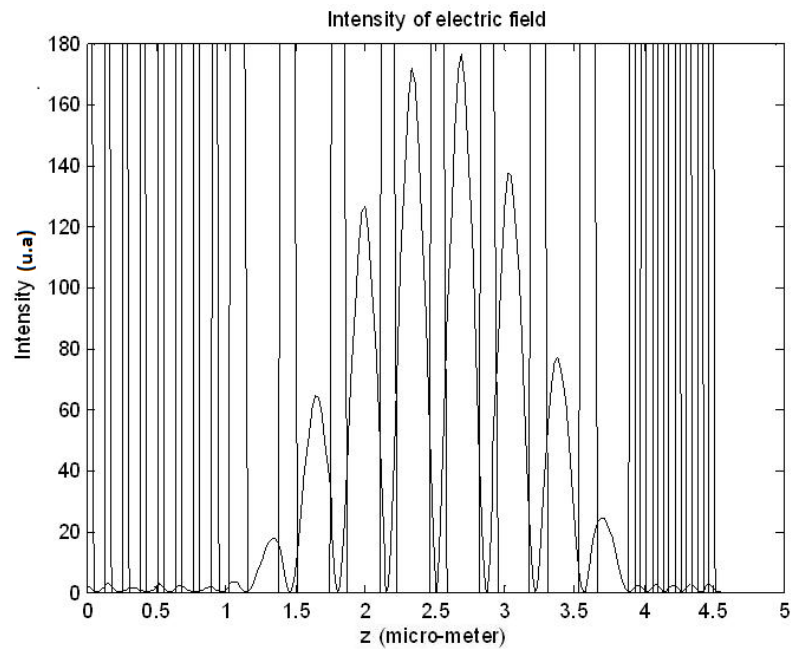

Fig. 8: Variation of the electric field intensity, versus the thickness $(\mu \mathrm{m})$ of hybrid photonic structure [Bragg_1: 16 layers $\left(\mathrm{n}_{\mathrm{H}}=3\right.$ and $\left.\mathrm{n}_{\mathrm{L}}=1.45\right) / \mathrm{CP}: 16$ layers $\left(\mathrm{n}_{\mathrm{H}}=2.3\right.$ and $\left.\mathrm{n}_{\mathrm{L}}=1\right),\left(\lambda_{0} / 2\right) /$ Bragg_2: 16 layers $\left(\mathrm{n}_{\mathrm{H}}=3.134\right.$ and $\mathrm{n}_{\mathrm{L}}=3$ ]

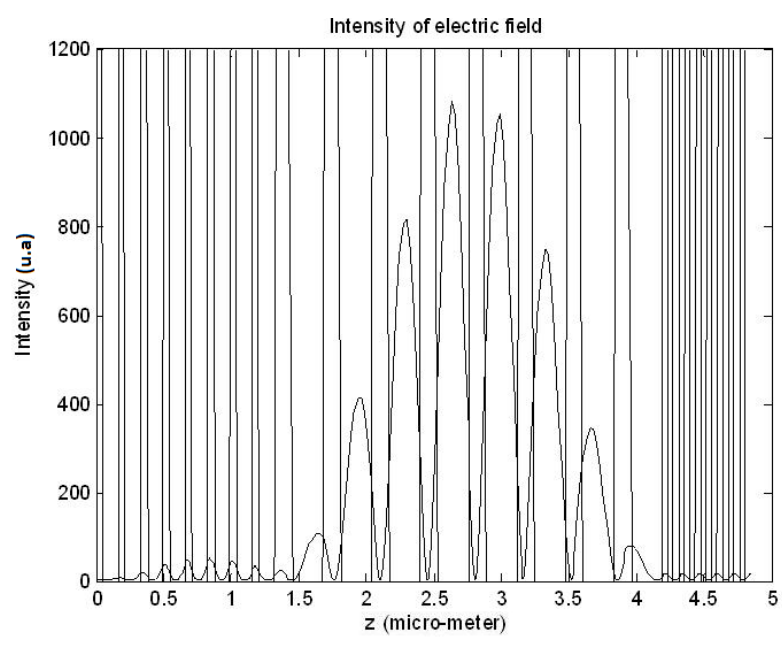

Fig. 9: Variation of the electric field intensity, versus the thickness $(\mu \mathrm{m})$ of hybrid photonic structure [Bragg_1: 16 layers $\left(\mathrm{n}_{\mathrm{H}}=3.134\right.$ and $\left.\mathrm{n}_{\mathrm{L}}=1\right) / C P: 16$ layers $\left(\mathrm{n}_{\mathrm{H}}=2.3\right.$ and $\left.\mathrm{n}_{\mathrm{L}}=1\right),\left(\lambda_{0} / 2\right) /$ Bragg_2: 16 layers $\left(\mathrm{n}_{\mathrm{H}}=3.134\right.$ and $\mathrm{n}_{\mathrm{L}}=3$ ] 


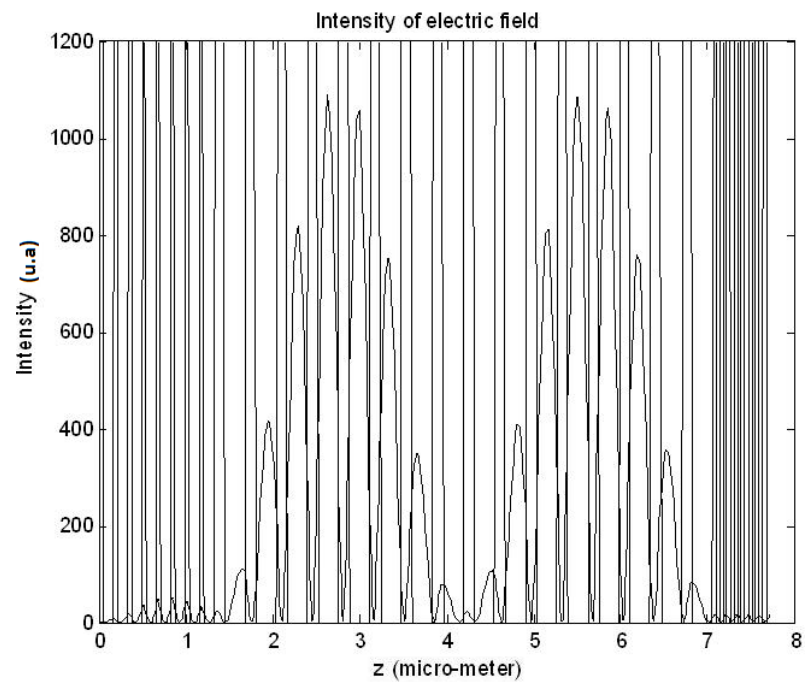

(a)

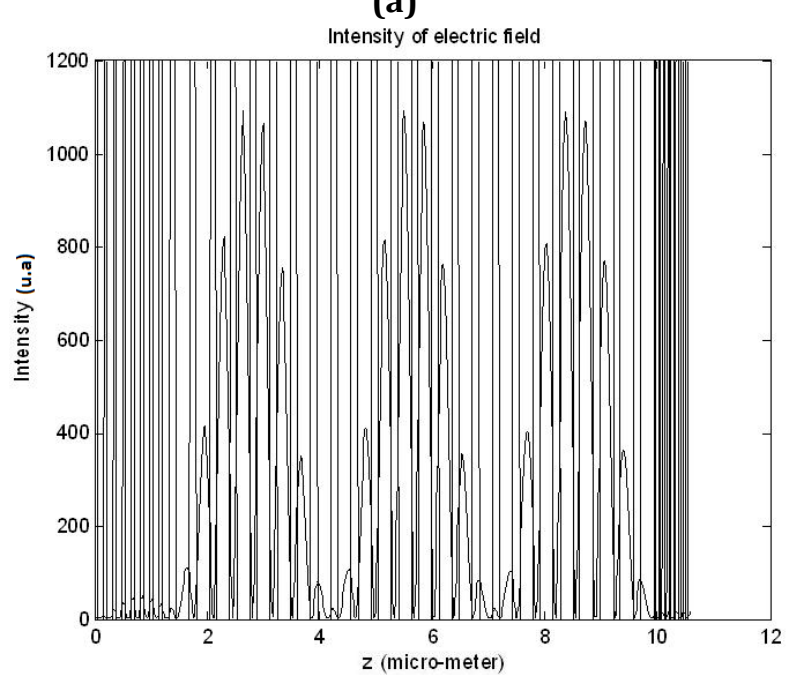

(b)

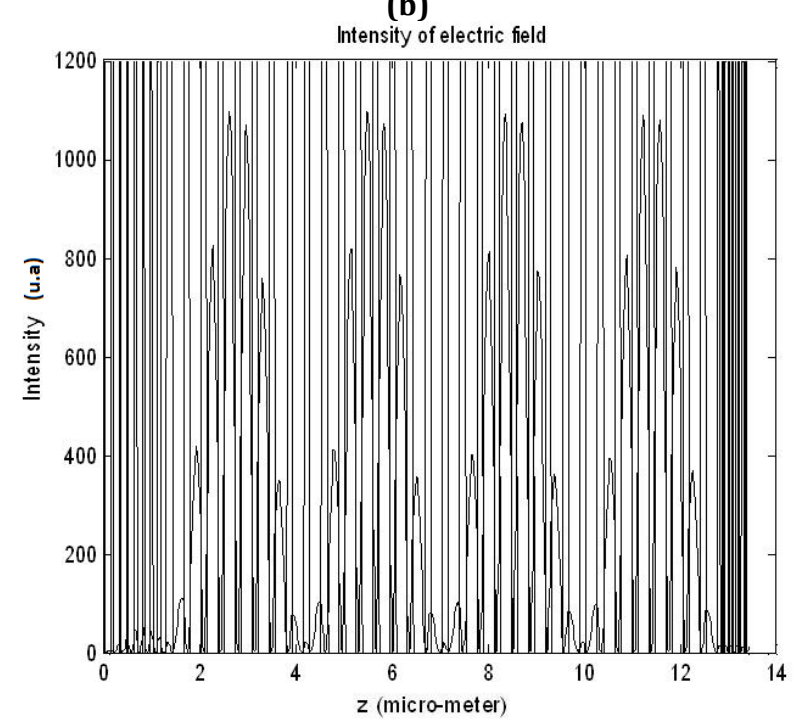

(c)

Fig. 10: Variation of the electric field intensity, versus the thickness $(\mu \mathrm{m})$ of hybrid photonic structure [Bragg_1: 16 layers $\left(\mathrm{n}_{\mathrm{H}}=3.134\right.$ and $\left.\mathrm{n}_{\mathrm{L}}=1\right) / \mathrm{CP}\left(\mathrm{n}_{\mathrm{H}}=2.3\right.$ and $\left.\mathrm{n}_{\mathrm{L}}=1\right)$,

$\left(\lambda_{0} / 2\right) /$ Bragg_2: 16 layers $\left(\mathrm{n}_{\mathrm{H}}=3.134\right.$ and $\mathrm{n}_{\mathrm{L}}=3$ ] for: (a) 32 layers of the PC, (b) 48 layers of the PC and (c) 64 layers of the PC

Now we fix the number of layers of the PC to16, and we study the effect of the number of layers in the
Bragg_2 mirror on the electric field intensity. So it is clear that the optimal value of the number of layers to obtain the maximum electric field intensity is 12 as shown by the Fig. 11. While keeping this number of layers, the same result was obtained for the Bragg_1 mirror which the optimal number of layers is 10 to obtain the higher electric field intensity in the PC (Fig. 12); also here we have seen that for any number of layers of Bragg_1 the resonance peak position are the same.
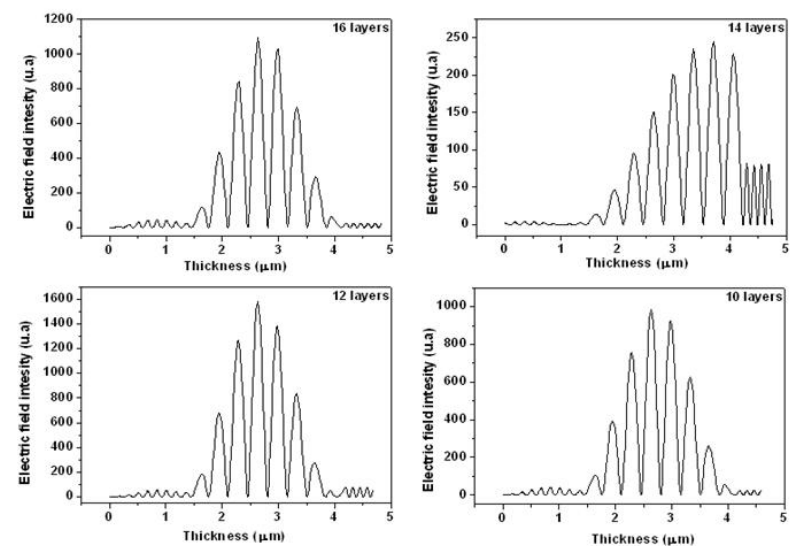

Fig. 11: Variation of the electric field intensity, versus the number of layers of Bragg_2 and the thickness $(\mu \mathrm{m})$ of the hybrid photonic structure [Bragg_1: 16 layers $\left(\mathrm{n}_{\mathrm{H}}=3.134\right.$ and $\left.\mathrm{n}_{\mathrm{L}}=1\right) / \mathrm{CP}: 16$ layers $\left(\mathrm{n}_{\mathrm{H}}=2.3\right.$ and $\left.\mathrm{n}_{\mathrm{L}}=1\right)$, $\left(\lambda_{0} / 2\right) /$ Bragg_2: $\left(\mathrm{n}_{\mathrm{H}}=3.134\right.$ and $\left.\mathrm{n}_{\mathrm{L}}=3\right]$
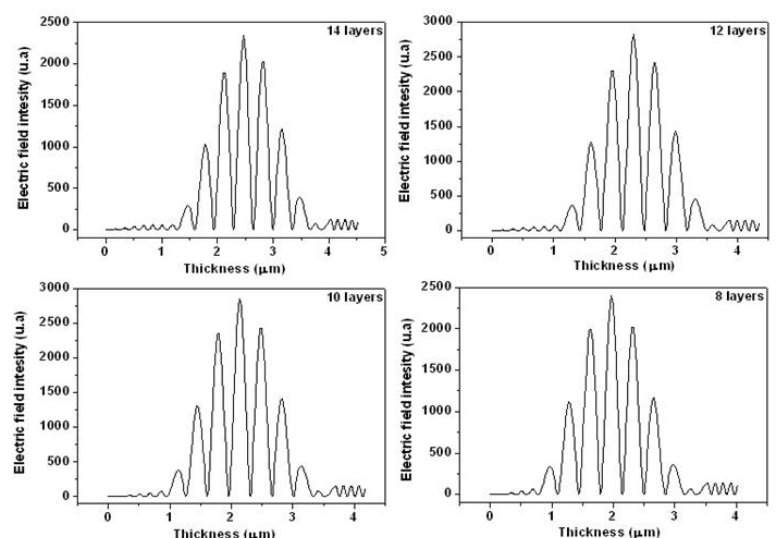

Fig. 12: Variation of the electric field intensity, versus the number of layers of Bragg_1 and the thickness $(\mu \mathrm{m})$ of the hybrid photonic structure [Bragg_1 $\left(\mathrm{n}_{\mathrm{H}}=3.134\right.$ and $\left.\mathrm{n}_{\mathrm{L}}=1\right) /$ CP: 16 layers $\left(\mathrm{n}_{H}=2.3\right.$ and $\left.\mathrm{n}_{\mathrm{L}}=1\right),\left(\lambda_{0} / 2\right) /$ Bragg_2: 12 layers $\left(\mathrm{n}_{\mathrm{H}}=3.134\right.$ and $\left.\mathrm{n}_{\mathrm{L}}=3\right]$

\subsection{Effects of the layers thickness}

In this section we investigate the effects of the layers thickness of the PC on the electric field intensity. In the last section it has been shown that the maximal value of this intensity (Fig. 12) is obtained by this hybrid structure: Bragg_1 10 layers $\left(n_{H}=3.134\right.$ and $\left.n_{L}=1\right) /$ PC: 16 layers $\left(n_{H}=2.3\right.$ and $\left.n_{L}=1\right)$, $\left(\lambda_{0} / 2\right) /$ Bragg_2: 12 layers $\left(n_{H}=3.134\right.$ and $\left.n_{L}=3\right)$.

At the present the thicknesses of the two materials which constitute the PC satisfy the condition: $n_{L} d_{L}=n_{H} d_{H}=\lambda_{0} / 4$, where $\lambda_{0}=0.5 \mu \mathrm{m}$. In this case, high electric field intensity value in the PC was obtained as shown in the Fig. 13. Therefore if we 
replace the PC by another one with optical thickness equal to $\lambda_{0} / 4$ we can also increase the electric field intensity (i.e. we enhance, localize and confine this field) in the thickness of the middle structure. The optical thickness $\lambda_{0} / 4$ is an optimal value to obtain high electric field intensity. So if we select three successive Bragg mirrors and we vary the materials that constitutes every structure we can enhance and localize (confine) the electric field in the layers of the middle structure and attenuate it in the structures of sides.

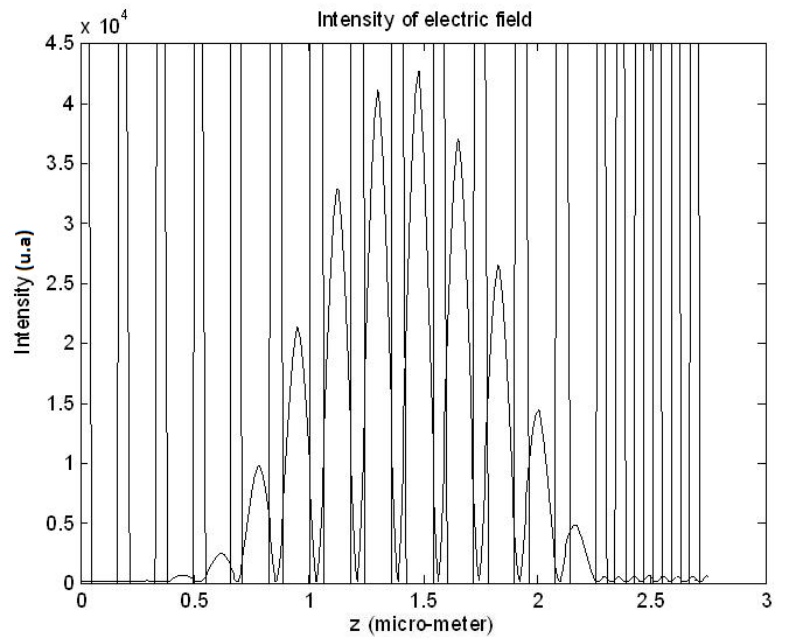

Fig. 13: Variation of the electric field intensity, versus the thickness $(\mu \mathrm{m})$ of hybrid photonic structure [Bragg_1: 10 layers $\left(\mathrm{n}_{\mathrm{H}}=3.134\right.$ and $\left.\mathrm{n}_{\mathrm{L}}=1\right) / C P: 16$ layers $\left(\mathrm{n}_{\mathrm{H}}=2.3\right.$ and $\left.\mathrm{n}_{\mathrm{L}}=1\right),\left(\lambda_{0} / 4\right)$ /Bragg_2: 12 layers $\left(\mathrm{n}_{\mathrm{H}}=3.134\right.$ and $\mathrm{n}_{\mathrm{L}}=3$ ]

\section{Conclusions}

The electric field intensity spectra in onedimensional hybrid Bragg mirrors/photonic crystal was analyzed by using the Transfer Matrix Method. It's demonstrated that if the contrast of refractive index of the first Bragg mirror (Bragg_1) is increased and the one of the second Bragg mirror (Bragg_2) is decreased, the electric field can be enhanced and localized (confine) in the PC, and disappeared in the Bragg mirrors. In addition we have noted that by varying the layers number of each structure that constitutes the hybrid photonic system, the electric field intensity increases more.

Eventually high enhancement and localization of electric field through the middle photonic structure was founded when we changed the PC by a Bragg mirror constituted by the same materials of this crystal. These optical performances in visible spectral range open a way toward the optical memory devices and increase the efficiency of nonlinear optical interactions.

\section{Acknowledgment}

The authors would like to thank the Deanship of the Scientific Research of University of Hail, Saudi Arabia for funding and supporting this research project (150161).

\section{Nomenclature}

TMM: Transfer Matrix Method.

BM: Bragg mirrors.

TE: Transverse-Electric polarization.

PC: Photonic crystals.

1D: One-Dimensional.

L: Low.

$H$ : High.

$\mathrm{TiO}_{2}$ : Titanium dioxide.

$\mathrm{SiO}_{2}$ : Silicon dioxide.

$\lambda_{0}$ : Reference wavelength.

$n$ : Refractive indices.

$d$ : Layers thicknesses.

$E_{0}^{+}$: Incident wave.

$E_{0}^{-}$: Reflected wave.

$E_{m+1}^{+}$: Transmitted wave after m layers.

$C_{j}$ : Transfer Matrix.

$C_{p r}$ : Propagation Matrix.

$C_{\text {int }}$ : Interface Matrix.

$t_{j}$ : Fresnel transmission coefficient.

$r_{j}$ : Fresnel reflection coefficients.

$j^{\text {th }}$ : Layer number $\mathrm{j}$.

$K$ : Wave number.

$\lambda$ : Wavelength.

\section{References}

Cheng DK (1993). Fundamentals of engineering electromagnetics. Addison-Wesley, USA.

Joannopoulos JD, Meade RD, Winn J (1995). Photonic crystals: Molding the flow of light. Princeton University, Princeton, New Jersey, USA.

Johnson SG, Joannopoulos JD (2002). Photonic crystals: The road from theory to practice. Kluwer, Boston, USA.

Kittel C and Holcomb DF (1967). Introduction to solid state physics. American Journal of Physics, 35(6): 547-548.

Lannebère S and Silveirinha MG (2015). Optical meta-atom for localization of light with quantized energy. Nature Communications, 6:8766.

Loncar M, Scherer A and Qiu Y (2003). Photonic crystal laser sources for chemical detection. Applied Physics Letters, 82(26): 4648-4650.

Sajeev J (1997). Photonics: Frozen light. Nature 390(6661): 661-662.

Topolancik J, Bhattacharya P, Sabarinathan J and Yu PC (2003). Fluid detection with photonic crystalbased multichannel waveguides. Applied Physics Letters, 82(8): 1143-1145.

Trofimov VA, Tereshin EB and Fedotov MV (2003). On the possibility of light energy localization in a nonlinear photonic crystal. Optics and Spectroscopy, 95(1): 106-109.

Vuckovic J, Englund D, Fattal D, Waks E and Yamamoto Y (2006). Generation and 
manipulation of nonclassical light using photonic crystals. Physica E: Low-Dimensional Systems and Nanostructures, 32(1): 466-470.
Wiersma DS, Bartolini P, Lagendijk A and Righini R (1997). Localization of light in a disordered medium. Nature, 390(6661): 671-673.

Yeh P and Yariv A (1984). Optical waves in crystals. A Wiley-Interscience publication, New York. 